\title{
Effects of different stocking densities on the trachea barrier function and its plasma metabolic profile of finishing broilers
}

\section{Yuanyuan Wang}

Zhejiang University

Dianchun Wang

Zhejiang University

Jiangshui Wang

Zhejiang University

Kaixuan Li

Zhejiang University

Chianning Heng

Zhejiang University

Lei Jiang

Zhejiang University

Chenhao Cai

Zhejiang University

Xiuan Zhan ( $\square$ xazan@zju.edu.cn )

https://orcid.org/0000-0001-6848-058X

\section{Research}

Keywords: stocking density, broiler, immunity, trachea barrier, metabolomics

Posted Date: May 22nd, 2020

DOI: https://doi.org/10.21203/rs.3.rs-28979/v1

License: (1) (1) This work is licensed under a Creative Commons Attribution 4.0 International License. Read Full License 


\section{Abstract}

Background The experiment was conducted to evaluate the effects of different stocking densities on the trachea barrier and its plasma metabolic profile of finishing broilers.

Methods One thousand four hundred and forty 22-day-old Lingnan Yellow feathered broilers were randomly allotted to five different stocking density groups $\left(8,10,12,14\right.$, and 16 birds per $\left.\mathrm{m}^{2}\right)$. Each group consisted of three replicates.

Results A markedly increased in IL-1 $\beta$ and IL-10 concentrations were observed in 16 birds per $\mathrm{m}^{2}$ treatment group than the groups of 8 and 10 birds per $\mathrm{m}^{2}(P<0.05)$, but there was no significant difference in IL-4 content among five treatments $(P>0.05) .16$ birds per $\mathrm{m}^{2}$ (high stocking density group, HSD) treatment group significantly increased the trachea mucosa thickness than 10 birds per $\mathrm{m}^{2}$ (control group, CSD). HSD group exhibited lower expression of claudin 1 and higher muc2 and caspase 3 than those in the CSD group. In addition, a total of 10 metabolites were identified at higher $(P<0.05)$ concentrations, while 7 metabolites were identified at lower $(P<0.05)$ concentrations in the HSD treatment as compared with the CSD group. Most of these potentially diagnostic biomarkers were involved in substance synthesis and energy metabolism. Further metabolic pathway analysis revealed that pentose and glucuronate interconversions along with pentose phosphate pathway were the most relevant and critical pathway identified from these two groups. The activated pathway may partially interpret the metabolic level to alleviate the adverse effects of stress which caused by high-stocking density in broilers.

Conclusions In summary, this study can improve our understanding of the harmful effects of highstocking density on the trachea barrier and identify two metabolic pathways that may be associated with high-stocking density-induced metabolic disorders in broilers.

\section{Background}

Stocking density of broiler is usually expressed either as the number of housed birds or as the live weight $(\mathrm{kg})$ per $\mathrm{m}^{2}$ floor space at depopulation or at the end of growing period [1]. In addition, stocking density is believed to have critical implications for poultry production [2]. Increasing stocking density can yield higher profits per unit space, hence increasing stocking density appropriately is expected in poultry production. However, excessive high-stocking density can cause a series of negative consequences for poultry production and welfare [3, 4]. For broilers, negative consequences including decreased broiler growth performance, feed utilization, carcass traits and immunity [5], increased physiological and oxidative stress and induced intestinal mucosal injury $[6,7]$.

Indeed, for crowded birds, an increase of the litter temperature due to the higher content of moisture and nitrogen, resulting in the dissipation of metabolic heat increase, which enhances the microbial activities [1]. As the litter quality will affect the environmental situation of the birds by dust levels, air humidity and 
ammonia levels, which in turn can lead to respiratory problems $[1,8]$. The respiratory tract is the first position of organisms to contact inhaled pathogenic microorganisms [9]. The mucous membrane of respiratory tract forms the first line of defense against the invasion of exotic pathogenic microorganisms. Therefore, the tracheal mucosal immune function plays an important role in resistance to the adverse stimulus [10].

Metabolomics is an emerging research area which can quantitatively measure a series of small molecular metabolites in biological samples using high-throughput approaches [11]. Identification and integrative analysis of these metabolites could enable a comprehensive characterization of metabolism at the molecular and cellular levels under a given set of physiological conditions [12]. Therefore, metabolomics analysis can provide novel insights into metabolic status alterations of biological systems, uncovering nutritional intervention mechanisms through metabolic pathways integrated with related metabolites [13]. Many researches have been reported on growth performance, antioxidant capability and physiological stress. Most of these studies, however, have evaluated only a narrow range of stocking densities. Moreover, majorities of these studies were not always conclusive and had produced variable conclusions. So far, there is little literature regarding the stocking density used Gas Chromatography Tandem Time-of-Flight Mass Spectrometry (GC-TOF/MS). In the current study, the effects of five stocking densities, ranging from 8 to 16 birds per $\mathrm{m}^{2}$, was thereby conducted to investigate trachea barrier function and identify the metabolic phenotype associated with high-stocking density that could be linked to growth and health status in broilers.

\section{Materials And Methods}

\section{Experiment birds and treatment}

The experiment birds and treatment was conducted in accordance with our previously described methods [14]. One thousand five hundred and thirty 1-day-old Lingnan Yellow broiler chickens were obtained from a local commercial hatchery. During the whole experiment, the birds were housed in 15 concrete floor rooms $\left(8 \mathrm{~m}^{2}\right)$ bedded with $6 \mathrm{~cm}$ rice hulls. Pre-experiment lasted for 21 days and during this period all birds were reared at the same stocking density $\left(12.75 \mathrm{birds} / \mathrm{m}^{2}\right)$. Sick and weak chicks observed during this period were removed. On day 22 , one thousand four hundred and forty broilers with a similar body weight were randomly allotted into 5 treatments, each with three replicates. Meanwhile, all rooms were filled with new rice hulls. The stocking densities of these 5 treatments were $8,10,12,14$, and 16 birds per $\mathrm{m}^{2}(64,80,96,112$, and 128 birds per room, respectively). The birds had free access to feed and water throughout the experimental period with light-dark cycles per day. During the experiment, all rooms were environmentally controlled at $25^{\circ} \mathrm{C}$. The relative humidity was maintained at $55 \pm 5 \%$. A corn-soybean meal basal diet was formulated to meet the nutrient requirement reference to the NRC (1994) (Table 1).

\section{Sampling and processing}


At the age of 50 days old, 12 male broilers (4 birds per replication) were randomly selected from each treatment. Feed was removed 12 hours before slaughter, while water was available. Blood samples by the vein under the wing ( $5 \mathrm{~mL} /$ bird) were immediately collected into a coagulant tube and centrifuged at $3000 \mathrm{rpm} / \mathrm{min}$ for $10 \mathrm{~min}$ at $4^{\circ} \mathrm{C}$ to obtain serum. Then the birds were slaughtered to obtain trachea. All samples were frozen directly in liquid nitrogen and stored at $-80^{\circ} \mathrm{C}$ for further analysis.

\section{Trachea morphology}

Trachea samples were embedded in paraffin wax after dehydration and sectioned at $5 \mu \mathrm{m}$ on a rotary microtome. The sections were further stained with haematoxylin and eosin [H\&E] for morphological analysis and digital images were obtained under a light microscope [Olympus].

\section{Relative protein expression by western blot}

The protein expression of claudin1, muc2 and caspase 3 in the trachea was determined by western blot. Total protein extraction was performed using T-PER Tissue Protein Extraction Reagent (Thermo Pierce, 78510), protein quantification was then performed using the BCA Quantitation Kit. After SDS-PAGE and membrane transfer, T-TBS (containing $5 \%$ non- fat dry milk or BSA) was added to the membrane for blocking at room temperature for $1 \mathrm{~h}$. The antibody (1:100) was then added and incubated overnight at $4^{\circ} \mathrm{C}$, followed by washing the membrane. Secondary antibody [Goat anti-Mouse IgG $(H+L)$ ] was added and incubated at room temperature for $1 \mathrm{~h}$, and then washed. SuperSignal ${ }^{\circledR}$ West Dura Extended Duration Substrate was used for western blot detection. The optical density of the bands was analyzed using Image $\mathrm{J}$ software. $\beta$-actin was used as an internal control and was found to exhibit no difference between groups. The relative abundance of each target protein was expressed as the ratio of target protein/ $\beta$-actin.

\section{Determination of immune status}

The commercial kits for all the cytokine indexes were purchased from Nanjing Jiancheng Bioengineering

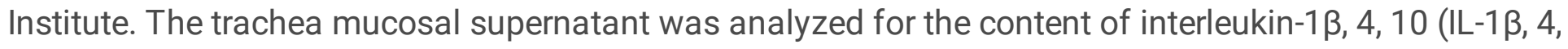
10). The procedures were carried out according to the description of the assay kits.

\section{Sample Preparation for GC-TOF/MS Analysis}

The samples were prepared for metabolomics analysis as described previously [15]. Briefly, $100 \mu \mathrm{L}$ plasma was added with $350 \mu \mathrm{L}$ of extraction liquid (methanol:acetonitrile:water $=2: 2: 1$, volume ratio) and $20 \mu \mathrm{L} \mathrm{L}$-2-chlorophenylalanine. The sample mixture was maintained at $4^{\circ} \mathrm{C}$ for 30 min and subsequently centrifuged at $13,000 \mathrm{rpm}$ for $15 \mathrm{~min}$. The supernatant $(400 \mu \mathrm{L})$ was collected into a $1.5 \mathrm{~mL}$ tube, and then transferred to a glass vial and dried in a vacuum. Methoxyamine salt reagent (methoxyamine hydrochloride, dissolved in pyridine $20 \mathrm{mg} / \mathrm{mL}$ ) was added and the mixture incubated in an oven at $80^{\circ} \mathrm{C}$ for 30 min, then derivatized using N, O Bis (trimethylsilyl) trifluoroacetamide (BSTFA), incubated for 1 hour at $70^{\circ} \mathrm{C}$. The final mixture was strongly vortexed for $1 \mathrm{~min}$ and used for analysis [16]. 


\section{GC-TOF/MS Analysis}

As our previously described methods [17], the derivatized samples were analyzed using an Agilent 7890 GC system equipped with a Pegasus 4D TOFMS (LECO, St. Joseph, MI) with a DB-5MS capillary column (30 m $\times 250 \mu \mathrm{m}$ inner diameter, $0.25 \mu \mathrm{m}$ film thickness coated with $95 \%$ dimethyllpolysiloxane crosslinked with $5 \%$ diphenyl under the following conditions: initial temperature was kept at $80^{\circ} \mathrm{C}$ for 12 seconds, increased to $180^{\circ} \mathrm{C}$ at a rate of $10^{\circ} \mathrm{C} / \mathrm{min}$, to $240^{\circ} \mathrm{C}$ at a rate of $5^{\circ} \mathrm{C} / \mathrm{min}$, and further to $290^{\circ} \mathrm{C} \mathrm{C}$ at a rate of $20^{\circ} \mathrm{C} / \mathrm{min}$ and maintained for $11 \mathrm{~min}$. One $\mu \mathrm{L}$ of sample solution was injected with helium as the carrier gas at a flow rate of $1 \mathrm{~mL} /$ minute. The temperatures of transfer line and ion source were $245^{\circ} \mathrm{C}$ and $220^{\circ} \mathrm{C}$ respectively. The MS data were acquired with a mass-to-charge ratio $(\mathrm{m} / \mathrm{z})$ range of 20 600 in a full-scan mode.

\section{GC-TOF/MS Data Acquisition and Processing}

The raw peaks extraction, data baselines filtering and calibration, peak alignment, deconvolution analysis, peak identification, and integration of the peak area were operated using the Chroma TOF4.3X software (LECO) and LECO-Fiehn Rtx5 database. The peak identification was tested using the RI (retention time index) method. The missing values of the original data were filled by half of the minimum value via a numerical simulation method. Noise removal was performed based on an interquartile range to filter data and then normalized by area normalization methods. The SIMCA14.1 software package (Umetrics, Umea, Sweden) was used for multivariate variable pattern recognition analysis: principal component analysis (PCA), partial least squares discriminant analysis (PLS-DA), and orthogonal partial least-squares discriminant analysis (OPLS-DA). PCA was used to show the internal structure of the data and display the similarity and difference. OPLS-DA was applied to obtain a higher level of group separation and better explain the variables. To evaluate the predictive ability and fitting level of the model, the parameters $R^{2} Y$ and $Q^{2}$ were applied. The metabolites responsible for differentiating two groups were filtered with the following requirements: variable importance in the projection (VIP) $>1$ and $P$-values of 0.05 (threshold) with 95\% Hotelling's T-squared ellipse [17].

\section{Statistical Analysis}

The data was examined by one-way analysis of variance (ANOVA) using IBM SPSS 19.0 and expressed as means with SEM derived from the ANOVA error mean square. Differences between means were examined through Duncan's multiple range tests using $P<0.05$. Moreover, Student's t-test was used to profile metabolite differences between two groups.

\section{Results}

\section{Immune status}

As shown in Table 2, with the increase of stocking density from 8 to 16 bird per $\mathrm{m}^{2}$, a markedly increased IL-1 $\beta$ and IL-10 concentrations were observed $(P<0.05)$, whereas there was no significant difference in IL- 
4 content $(P>0.05)$. Compared with 8,10 birds per $\mathrm{m}^{2}$ groups, 16 birds per $\mathrm{m}^{2}$ group had higher concentrations of IL-1 $\beta$ and IL-10 $(P<0.05)$.

\section{Immune system and trachea barrier function}

Digital images of H\&E-stained sections indicated that the trachea structure of the 10 birds per $\mathrm{m}^{2}$ (control group, CSD) group presented normal trachea morphology. However, 16 bird per $\mathrm{m}^{2}$ (high stocking density group, HSD) treatment group significantly increased the trachea mucosa thickness. In addition, the mucosal surface was fragmented with thickened submucosa and increased goblet cells as well as more ciliated cells were discovered under high magnification (Fig. 1a). To further investigate the effect of HSD on trachea physical barrier function, the relative protein expression of claudin1, muc2 and caspase 3 in trachea tissue were examined. HSD treatment group significantly reduced claudin 1 expression while increased muc2 and caspase3 expression compared with CSD (Fig. 1b, c).

\section{Metabolic differences of serum between 16 birds and 10 bird per $\mathrm{m}^{2}$ treatment groups}

The total GC-TOF-MS ion chromatogram of the HSD and CSD groups acquired by the UPLC-MS platform are presented in Figure 2. Following pretreatment and standardization using MZ mine 2.0 software, 425 integral peaks following extraction ion chromatography were detected in QC samples, and 629 peaks in test samples.

To explore the functional changes of the serum metabolome, the serum in each sample of HSD and CSD treatment groups was analyzed by multiple MS platforms. A principal component analysis was first performed to show a trend of intergroup separation on the scores plot (Fig. 3a), in which HSD treatment broilers were clearly separated from controls. This method also enabled detection and exclusion of any outliers, defined as observations located outside the $95 \%$ confidence region of the model. An OPLS-DA method was performed to better understand the different metabolic patterns. Figure $3 \mathrm{~b}$ showed significantly separated clusters between HSD (blue circle) and CSD (green square) treatment groups. The values for $\mathrm{R}^{2} \mathrm{X}$ and $\mathrm{Q}^{2}$ and the results of permutation tests indicated that the samples were of reasonable quality (Fig. 3c).

We determined those differentially expressed metabolites that played the greatest roles in separating the HSD and CSD treatment groups. The profile of potential biomarkers between the HSD and CSD groups was visualized by a volcano plot (Fig. 4). And a total of 17 biomarkers metabolites were filtered according to the standard with similarity $>600$, VIP $>1$ and $P<0.05$. These metabolites in serum mostly are lipids, amino acids, carbohydrates, organic acids, amines and others, involved in multiple biochemical processes, including 6-Phosphogluconic Acid, Threonic Acid, Xylitol, Benzoic Acid, Heptadecanoic Acid, Threonine 2, Halostachine 2, Indole-3-Acetic Acid, Dioctyl Phthalate, Salicin, Uric Acid, 2,3Dihydroxypyridine, Conduritol b Epoxide 2, Glucoheptonic Acid 1, 3-(1-Pyrazolyl)-L-Alanine, Cystine and 4Acetamidobutyric Acid (Table 3). In addition, a total of 10 metabolites were identified at higher $(P<0.05)$ concentrations while 5 metabolites were identified at lower $(P<0.05)$ concentrations in the HSD 
treatment group as compared to the CSD. Therein, four candidate metabolites (6-Phosphogluconic Acid, Threonic Acid, Salicin, 3-(1-Pyrazolyl)-L-Alanine) were selected as biomarkers, as they showed high sensitivity, specificity and accuracy in diagnosing broilers under high stocking density stress $(P<0.01)$, and further research is warranted to evaluate these biomarkers in practical applications and to elucidate the physiological mechanisms of high stocking density-induced metabolic disorder.

The current study not only investigated the different metabolites in serum between the HSD and CSD groups, but also pinpointed the pathways in which these metabolites were involved. A total of 9 enriched pathways exhibited impact values at the comprehensive level (Table 4). Among them, pentose and glucuronate interconversions, pentose phosphate pathway, as well as glycine serine and threonine metabolism had pathway impact values higher than 0.00 , which is the cutoff value for relevance. The impact values of these three metabolic pathways were $0.08,0.08$ and 0.05 , respectively. When the statistical $P$ values were further adjusted via hypergeometric testing for enrichment analysis, only pentose and glucuronate interconversions along with pentose phosphate pathway exhibited significant differences $(P<0.05)$, and these two metabolic pathways were thus characterized as the significantly relevant pathways (Fig. 5).

\section{Discussion}

The importance of animal welfare for European consumers and citizens has recently been confirmed by the European Union barometers [18]. The animal related products, especially food, to be produced with respect for the welfare of animal is drawn attention by more and more consumers $[19,20]$. It is also known that the increase of stocking density can reduces the bird welfare. Previous study in our lab found that stocking density of broilers up to 8 or $10 \mathrm{birds} / \mathrm{m}^{2}$ can prevent the negative effect on growth performance and welfare parameters in broilers [21].

Cytokines can influence the immune system and also are of importance within the innate immune system $[22,23]$. Cytokines are soluble, potent glycoproteins involved in the regulation of growth, immune cell activation, and the inflammatory and immune responses [24]. To further explore the mechanism of highstocking density in undermining trachea barrier function in broilers, trachea mucosal immunity under different conditions were analyzed and found that HSD group significantly increased the secretion of IL$1 \beta$ and IL-10 in the trachea. Cytokines can be pro-inflammatory or anti-inflammatory. IL $1 \beta$ is proinflammatory cytokines that activate additional cytokine cascades and increase organ damage, which plays a role in orchestrating the physiological and behavioral adjustments during sickness [25]. However, IL1 receptor antagonist and IL10 act as anti-inflammatory cytokines that have protective effects [26]. IL10 deactivates macrophages, which in turn decreases the production of cytokines by $T$ cells [27], it has broad anti-inflammatory effects and acts through suppression of pro-inflammatory cytokine production [28]. In addition to the role of IL-10 in positive feedback loops involving cytokines, it is known to relay negative feedback signals that dampen the activated immune system after an inflammatory trigger [26, 29]. Excessive production of pro-inflammatory cytokines are followed by the development of immune- 
mediated inflammation and fibrosis of multiple organs [30]. However, the increase of anti-inflammatory cytokines IL-10 in HSD treatment prevent excessive inflammation and maintain immune tolerance.

Tracheal defense function mainly depends on the integrity of its mucosa and the secretion of protective proteins [31]. To the best of our knowledge, most of the previous experiment reported that increasing stocking density linearly decreased intestinal barrier function [32], but there is rarely any report on the effects of high-stocking density on trachea barrier function. In this study, the tracheal structure of CSD group had normal morphology, whereas, after high-stocking density stimulation of 29 days, HSD treatment group was damaged seriously with high mucosal thickness and inflammatory cell infiltration. Barrier function is often determined by tight junction integrity of epithelial layers because tight junction plays an important role in sealing the apical gaps [33]. The decreased expression of tight junction-related genes and proteins has been considered a molecular evidence for impaired barrier functions [34]. Therefore, we measured selected tight junction-related protein expression in the trachea mucosa. Claudin1 has been identified as an integral membrane protein localizing at tight junction, and introduced claudin1 can form tight junction-like networks in fibroblasts [35]. An observation for significantly reduced claudin-1 expression caused by HSD treatment in this experiment. The epithelium of the respiratory mucosa provides a barrier against injurious luminal agents, including bacteria, enzymes, and toxins [36]. The present study found that HSD markedly increased muc2 protein expression. As MUC gene transcription has been shown to be induced upon exposure of the airways to a number of substances that induce mucus secretion, such as endotoxin, $\mathrm{SO}_{2}$, and allergens [37]. The normal respiratory epithelium is coated with mucus, which provides a variety of protective functions. However, mucus hypersecretion is a key feature of allergic asthma and is associated with the clinical symptoms, airway obstruction, and mortality of the disease [36]. In addition, caspase activation is an essential step in programmed cell death (apoptosis) [38]. The current study suggested that HSD could significantly increased caspase3 expression compared to CSD group.

To date, however, there have been no metabolomics studies of broilers affected by high-stocking density. A previous research applied metabolomics approach to investigate the altered metabolic pattern in sera from patients with asthma and sought to identify the mechanism underlying asthma and potential biomarkers [39]. In our present study, those differentially expressed metabolites are primarily involved in the matter synthesis and energy metabolism. From the identified metabolites, 10 metabolites were found at higher levels whereas 7 metabolites were found at lower levels in the HSD group. Specifically, threonic acid is a major breakdown product of ascorbic acid used as a food additive, which could be very profitable for the health status of the host [40]. Xylitol is a five-carbon polyalcohol, pentitol, which has many advantages as a food ingredient and is widely distributed in nature [41]. In the human body, xylitol is both an endogenous metabolite of the uronic acid cycle and an exogenous source of energy [42]. Xylitol has application and potential for food and pharmaceutical industries. It is a functional sweetener as it has prebiotic effects which can reduce blood glucose, triglyceride, and cholesterol level [43]. Threonine is a major component of plasma globulin in poultry, rabbits and humans [44]. Bhargava et al. (1971) reported that antibody titers of chicks increased as dietary threonine increased [45]. In addition, a 
decrease in plasma IgG in sows was alleviated by feeding a threonine supplemented diet [46]. Taken together, these data suggest a role for threonine in immune function. Moreover, including organic acids (Glucoheptonic Acid and Acetamidobutyric Acid) and amino acids (Alanine and Cystine) together with other regulating factors may could be very profitable for the health status of the host. Accordingly, we deduced that chronic stress caused by high-stocking density will induce alterations of the levels of serum metabolites to alleviate the adverse effects of stress.

Herein, these low molecular weight metabolites were involved in multiple pathways and reactions, including pentose and glucuronate interconversions along with pentose phosphate pathway. It is well know that pentose and glucuronate interconversions has major biological significance for detoxification and excretion of metabolites [47]. A serum metabolic study revealed that D-glucuronic acid was associated with the pentose and glucuronate interconversions pathway[48]. The pentose phosphate pathway plays a crucial role in the host-parasite relationship. It maintains a pool of NADPI-i, zohich serves to protect against oxidant stress and which generates carbohydrate intermediates used in nucleotide and other biosynthetic pathways [49]. Thereupon, pentose phosphate pathway serves to convert glucose 6-phosphate to ribose 5-phosphate, which is used in nucleotide biosynthesis. In some systems, it also provides other important phosphorylated carbohydrates, such as erythrose 4-phosphate, which serves as a precursor in the synthesis of both aromatic amino acids and vitamins, as well as sedoheptulose 7-phosphate, an important component of some bacterial cell walls [50]. The other key product of the pathway is NADPH, which serves as a hydrogen donor in reductive biosynthesis, and plays an integral role in the defense against oxidative stress and detoxification of xenobiotics [49]. Collectively, considering the influential roles of pentose and glucuronate interconversions and pentose phosphate pathway in the body, it is speculated that the activation of these pathways could be, at least partially, responsible for the host to alleviate the adverse factors caused by high-stocking density.

\section{Conclusions}

Based on the evidences above, it can be concluded that with the increase of broiler density, the function of trachea barrier was impaired. In addition, metabolomics analysis revealed significant changes in the serum metabolite profiles of broilers in response to high-stocking density. The modified metabolites induced by high-stock density were predominantly connected with energy metabolism, in which pentose and glucuronate interconversions and pentose phosphate pathway were the most relevant and pivotal pathway. The results from this study uncovered the complex metabolic effects of high-stock density on broilers, which elucidate the potential mechanisms associated with the adverse effects of high-stock density.

\section{Declarations}

\section{Availability of data and materials}


All data generated or analyzed during this study are available from the corresponding author on reasonable reques.

\section{Ethics approval}

The project was conducted under the supervision of Zhejiang University Animal Care and Use Committee (Hangzhou, China), which has adopted animal care and use guidelines governing all animal use in experimental producers.

\section{Consent for publication}

Not applicable.

\section{Competing interests}

The authors declare that they have no competing interests.

\section{Funding}

The financial support provided by National Key R\&D Program of China (project: 2016YFD05005, Beijing, China), Zhejiang Province Key R\&D Program of China (project: 2018C02035, Hangzhou, China), China Agriculture Research System (project: CARS-42-G19, Beijing, China) are gratefully acknowledged.

\section{Author's contributions}

Yuanyuan Wang, Chenhao Cai and Xiuan Zhan designed and supervised the experiments. Yuanyuan Wang, Dianchun Wang, Jiangshui Wang, Kaixuan Li, Chianning Heng and Lei Jiang conducted the experiments. Yuanyuan Wang and Chenhao Cai performed the data measurements and statistical data analysis. Yuanyuan Wang and Xiuan Zhan wrote and revised the manuscript. All authors have read and approved the final manuscript.

\section{Acknowledgments}

We are grateful to Chenhao Cai for the assistance with the animal feed experiment.

\section{Abbreviations}

HSD, high stocking density group; CSD, control group; GC-TOF/MS, Gas Chromatography Tandem Timeof-Flight Mass Spectrometry; H\&E, haematoxylin and eosin; PCA, principal component analysis; PLS-DA, partial least squares discriminant analysis; OPLS-DA, orthogonal partial least-squares discriminant analysis; VIP, variable importance in the projection. 


\section{References}

1. Meluzzi A, Sirri F. Welfare of broiler chickens. Ital J Anim Sci. 2009;8(sup1):161-173. https://doi.org/10.4081/ijas.2009.s1.161.

2. Simsek U, Cerci I, Dalkilic B, Yilmaz O, Ciftci M. Impact of stocking density and feeding regimen on broilers: Chicken meat composition, fatty acids, and serum cholesterol levels. J Appl Poutry Res. 2009;18(3):514-520. https://doi.org/10.3382/japr.2008-00141.

3. Wang C, Liu Z, Xue J, Wang Y, Huang X, Wang Q. Effect of Stocking Density on Growth Performance, Feather Quality, Carcass Traits, and Muscle Chemical Component of Geese from 49 to 70 Days of Age. J Appl Poutry Res. 2019;28(4):1297-1304. https://doi.org/10.3382/japr/pfz097.

4. Mitchell M, Kettlewell P. Physiological stress and welfare of broiler chickens in transit: solutions not problems! Poult Sci. 1998;77(12):1803-1814. https://doi.org/10.1093/ps/77.12.1803.

5. Houshmand M, Azhar K, Zulkifli I, Bejo M, Kamyab A. Effects of prebiotic, protein level, and stocking density on performance, immunity, and stress indicators of broilers. Poult Sci. 2012;91(2):393-401. https://doi.org/10.3382/ps.2010-01050.

6. Li W, Wei F, Xu B, Sun Q, Deng W, Ma H, et al. Effect of stocking density and alpha-lipoic acid on the growth performance, physiological and oxidative stress and immune response of broilers. Asian Austral J Anim. 2019;32(12):1914. https://doi.org/10.5713/ajas.18.0939.

7. MASHALY MM, WEBB ML, YOUTZ SL, ROUSH WB, Graves $\mathrm{H}$. Changes in serum corticosterone concentration of laying hens as a response to increased population density. Poult Sci. 1984;63(11):2271-2274. https://doi.org/10.3382/ps.0632271.

8. Abudabos AM, Samara EM, Hussein EO, Al-Ghadi MaQ, Al-Atiyat RM. Impacts of stocking density on the performance and welfare of broiler chickens. Ital J Anim SCI. 2013;12(1):e11. https://doi.org/10.4081/ijas.2013.e11.

9. Pizzolla A, Nguyen TH, Smith JM, Brooks AG, Kedzierska K, Heath WR, et al. Resident memory CD8+ $T$ cells in the upper respiratory tract prevent pulmonary influenza virus infection. Sci Immunol. 2017;2(12):eaam6970. https://doi.org/10.1126/sciimmunol.aam6970.

10. Fan XX, Liu SQ, Liu GH, Zhao JP, Jiao HC, Wang XJ, et al. Vitamin A Deficiency Impairs Mucin Expression and Suppresses the Mucosal Immune Function of the Respiratory Tract in Chicks. Plos One. 2015;10(9). https://doi.org/10.1371/journal.pone.0139131.

11. Wang W, Li Z, Gan L, Fan H, Guo Y. Dietary supplemental Kluyveromyces marxianus alters the serum metabolite profile in broiler chickens. Food Funct.2018;9(7):3776-3787. https://doi.org/10.1039/c8fo00268a.

12. Fiehn 0 . Combining genomics, metabolome analysis, and biochemical modelling to understand metabolic networks. Int J Genomics. 2001;2(3):155-168. https://doi.org/10.1002/cfg.82. 
13. Ibero-Baraibar I, Romo-Hualde A, Gonzalez-Navarro CJ, Zulet MA, Martinez JA. The urinary metabolomic profile following the intake of meals supplemented with a cocoa extract in middle-aged obese subjects. Food Funct. 2016;7(4):1924-1931. https://doi.org/10.1039/c5fo01191d.

14. Cai CH, Zhao RX, Wang P, Wang JS, Li KX, Zhan XA, et al. Effects of different stocking densities on growth performance, antioxidant ability, and immunity of finishing broilers. Anim Sci J. 2019;90(4):583-588. https://doi.org/10.1111/asj.13148.

15. Dunn WB, Broadhurst D, Begley P, Zelena E, Francis-McIntyre S, Anderson N, et al. Procedures for large-scale metabolic profiling of serum and plasma using gas chromatography and liquid chromatography coupled to mass spectrometry. Nat Protoc. 2011;6(7):1060. https://doi.org/10.1038/nprot.2011.335.

16. Li Y, Guo Y, Wen Z, Jiang X, Ma X, Han X. Weaning Stress Perturbs Gut Microbiome and Its Metabolic Profile in Piglets. Sci Rep. 2018;8(1):18068. https://doi.org/10.1038/s41598-018-33649-8.

17. Wang Y, Xia L, Guo T, Heng C, Jiang L, Wang D, et al. Metabolic changes and physiological responses of broilers in the final stage of growth exposed to different environmental temperatures. Poult Sci. 2020;99(4):2017-2025. https://doi.org/10.1016/j.psj.2019.11.048.

18. Verdes S, Trillo Y, Pena Al, Herradon PG, Becerra JJ, Quintela LA. Relationship between quality of facilities, animal-based welfare indicators and measures of reproductive and productive performances on dairy farms in the northwest of Spain. Ital J Anim Sci. 2020;19(1):319-329. https://doi.org/10.1080/1828051X.2020.1743784.

19. Sanchez LS, Blanco-Penedo I, Munoz JMP, Perez CQ, Delgado JV, Vega-Pla JL. Welfare assessment at a Spanish Army Equine Breeding Centre. Ital J Anim Sci. 2020;19(1):137-146. https://doi.org/10.1080/1828051X.2019.1706433.

20. Dawkins MS, Donnelly CA, Jones TA. Chicken welfare is influenced more by housing conditions than by stocking density. Nature. 2004;427(6972):342-344. https://doi.org/10.1038/nature02226.

21. Cai CH, Zhao RX, Wang P, Wang JS, Li KX, Zhan XA, et al. Effects of different stocking densities on growth performance, antioxidant ability, and immunity of finishing broilers. Anim Sci J. 2019;90(4):583-588. https://doi.org/10.1111/asj.13148.

22. Wang $Y$, Wang $Y, X u H$, Mei X, Gong L, Wang B, et al. Direct-fed glucose oxidase and its combination with $\mathrm{B}$. amyloliquefaciens SC06 on growth performance, meat quality, intestinal barrier, antioxidative status, and immunity of yellow-feathered broilers. Poult Sci. 2018;97(10):3540-3549. https://doi.org/10.3382/ps/pey216.

23. Wilson D, Jackson T, Sapey E, Lord JM. Frailty and sarcopenia: the potential role of an aged immune system. Agening Res Rev. 2017;36:1-10. https://doi.org/10.1016/j.arr.2017.01.006.

24. Sinha S, Patil S, Jayalekshmy V, Satishchandra P. Do cytokines have any role in epilepsy? Epilepsy Res. 2008;82(2-3):171-176. https://doi.org/10.1016/j.eplepsyres.2008.07.018.

25. Pugh CR, Fleshner M, Watkins LR, Maier SF, Rudy JW. The immune system and memory consolidation: a role for the cytokine IL-1 $\beta$. Neurosci Biobehav R. 2001;25(1):29-41. https://doi.org/10.1016/s0149-7634(00)00048-8. 
26. Youn Y, Sung IK, Lee IG. The role of cytokines in seizures: interleukin (IL)-1 $\beta$, IL-1Ra, IL-8, and IL-10. Korean J Pediatr. 2013;56(7):271. https://doi.org/10.3345/kjp.2013.56.7.271.

27. Benner B, Scarberry L, Suarez-Kelly LP, Duggan MC, Campbell AR, Smith E et al. Generation of monocyte-derived tumor-associated macrophages using tumor- conditioned media provides a novel method to study tumor-associated macrophages in vitro. J Immunother Cancer. 2019;7. https://doi.org/10.1186/s40425-019-0622-0.

28. Li G, Bauer S, Nowak M, Norwood B, Tackenberg B, Rosenow F, et al. Cytokines and epilepsy. Seizure. 2011;20(3):249-256. https://doi.org/10.1016/j.seizure.2010.12.005.

29. Papadakis KA, Targan SR. Role of cytokines in the pathogenesis of inflammatory bowel disease. Annu Rev Med. 2000;51(1):289-298. https://doi.org/10.1146/annurev.med.51.1.289.

30. Yehudai-Ofir D, Henig I, Zuckerman T. Aberrant B cells, autoimmunity and the benefit of targeting $B$ cells in chronic graft-versus-host disease. Autoimmun Rev. 2020;19(4):102493. https://doi.org/10.1016/j.autrev.2020.102493.

31. Su Y, Wei H, Bi Y, Wang Y, Zhao P, Zhang R, et al. Pre-cold acclimation improves the immune function of trachea and resistance to cold stress in broilers. J Cell Physiol. 2019;234(5):7198-7212. https://doi.org/10.1002/jcp.27473.

32. Goo D, Kim J, Choi H, Park G, Han G, Kil D. Effect of stocking density and sex on growth performance, meat quality, and intestinal barrier function in broiler chickens. Poult Sci. 2019;98(3):1153-1160. https://doi.org/10.3382/ps/pey491.

33. Moretó M, Pérez-Bosque A. Dietary plasma proteins, the intestinal immune system, and the barrier functions of the intestinal mucosa. J Anim Sci.2009;87(14):E92-E100. https://doi.org/10.2527/jas.2008-1381.

34. Gilani S, Howarth G, Nattrass G, Kitessa S, Barekatain R, Forder R, et al. Gene expression and morphological changes in the intestinal mucosa associated with increased permeability induced by short-term fasting in chickens. J Anim Physiol An N. 2018;102(2):e653-e661. https://doi.org/10.1111/jpn.12808.

35. Inai T, Kobayashi J, Shibata Y. Claudin-1 contributes to the epithelial barrier function in MDCK cells. Eur J Cell Biol. 1999, 78(12):849-855. https://doi.org/10.1016/S0171-9335(99)80086-7.

36. Wills-Karp M. Trophic slime, allergic slime. Am J Resp Cell Mol. 2000, 22(6):637-639. https://doi.org/10.1165/ajrcmb.22.6.f190.

37. Jany B, Gallup M, Tsuda T, Basbaum C. Mucin gene expression in rat airways following infection and irritation. Biochem Bioph Res Co. 1991, 181(1):1-8. https://doi.org/10.1016/S0006-291X(05)81373-7.

38. Estrov Z, Thall PF, Talpaz M, Estey EH, Kantarjian HM, Andreeff M, et al. Caspase 2 and caspase 3 protein levels as predictors of survival in acute myelogenous leukemia. Blood. 1998, 92(9):30903097. https://doi.org/10.1182/blood.V92.9.3090.

39. Jung J, Kim SH, Lee HS, Choi G, Jung YS, Ryu D, et al. Serum metabolomics reveals pathways and biomarkers associated with asthma pathogenesis. Clin Exp Allergy. 2013;43(4):425-433. 
https://doi.org/10.1111/cea.12089.

40. Thomas M, Hughes R. A relationship between ascorbic acid and threonic acid in guinea-pigs. Food Chem Toxicol. 1983, 21(4):449-452. https://doi.org/10.1016/0278-6915(83)90101-1.

41. Mäkinen KK. Xylitol and oral health. Adv Food Res. 1979;25:137-158. https://doi.org/10.1016/S00652628(08)60236-0.

42. Ylikahri R. Metabolic and nutritional aspects of xylitol. Adv Food Res. 1979;25:159-180. https://doi.org/10.1016/s0065-2628(08)60237-2.

43. Ur-Rehman S, Mushtaq Z, Zahoor T, Jamil A, Murtaza MA. Xylitol: a review on bioproduction, application, health benefits, and related safety issues. Crit Rev Food Sci. 2015;55(11):1514-1528. https://doi.org/10.1080/10408398.2012.702288.

44. Tenenhouse HS, Deutsch H. Some physical-chemical properties of chicken y-globulins and their pepsin and papain digestion products. Immunochemistry. 1966;3(1):11-20. https://doi.org/10.1016/0019-2791(66)90277-1.

45. Bhargava K, Hanson R, Sunde M. Effects of threonine on growth and antibody production in chicks infected with Newcastle disease virus. Poult Sci. 1971;50(3):710-713. https://doi.org/10.3382/ps.0500710.

46. Cuaron JA, Chapple RP, Easter RA. Effect of lysine and threonine supplementation of sorghum gestation diets on nitrogen balance and plasma constituents in first-litter gilts. J Anim Sci. 1984, 58(3):631-637. https://doi.org/10.2527/jas1984.583631x.

47. Fang $H$, Zhang A, Yu J, Wang L, Liu C, Zhou X, et al. Insight into the metabolic mechanism of scoparone on biomarkers for inhibiting Yanghuang syndrome. Sci Rep. 2016;6:37519. https://doi.org/10.1038/srep37519.

48. Sun H, Zhang A-h, Song Q, Fang H, Liu X-y, Su J, et al. Functional metabolomics discover pentose and glucuronate interconversion pathways as promising targets for Yang Huang syndrome treatment with Yinchenhao Tang. Rsc Adv. 2018;8(64):36831-36839. https://doi.org/10.1039/c8ra06553e.

49. Barrett M. The pentose phosphate pathway and parasitic protozoa. Parasitol Today. 1997;13(1):1116. https://doi.org/10.1016/S0169-4758(96)10075-2.

50. Wood T. Physiological functions of the pentose phosphate pathway. Cell Biochem Funct. 1986;4(4):241-247. https://doi.org/10.1002/cbf.290040403.

\section{Tables}

Table 1. Composition and nutrient level of the basal diet (\% air-dry basis) 


\begin{tabular}{|c|c|c|c|}
\hline Items & Starter (1-21d) & Grower (22-42d) & Finisher (43-60d) \\
\hline \multicolumn{4}{|l|}{ Ingredients, \% } \\
\hline Corn & 58.00 & 35.50 & 33.50 \\
\hline Sorghum & - & 16.00 & 17.00 \\
\hline Barley & - & 10.00 & 13.00 \\
\hline Soybean meal & 34.00 & 19.70 & 15.70 \\
\hline Corn gluten meal & 2.00 & 5.80 & 5.30 \\
\hline DDGS $^{\mathrm{c}}$ & & 5.00 & 8.00 \\
\hline Lard & - & 4.00 & 3.70 \\
\hline Soybean oil & 1.80 & - & - \\
\hline $\mathrm{NaCl}$ & 0.40 & 0.30 & 0.30 \\
\hline $\mathrm{CaHPO}_{4}$ & 1.30 & 1.00 & 1.00 \\
\hline Limestone & 1.40 & 1.20 & 1.20 \\
\hline Zeolite & 0.10 & 0.50 & 0.30 \\
\hline Premix ${ }^{a}$ & 1.00 & 1.00 & 1.00 \\
\hline Total & 100.00 & 100.00 & 100.00 \\
\hline \multicolumn{4}{|c|}{ Nutrient levels ${ }^{\mathrm{b}}(\%)$} \\
\hline $\mathrm{ME}(\mathrm{MJ} / \mathrm{kg})$ & 12.10 & 12.93 & 12.92 \\
\hline $\mathrm{CP}$ & 22.94 & 21.63 & 19.63 \\
\hline Lys & 1.25 & 1.03 & 0.90 \\
\hline Met & 0.56 & 0.47 & 0.44 \\
\hline Met+Cys & 0.90 & 0.78 & 0.74 \\
\hline Calcium & 1.12 & 0.94 & 0.98 \\
\hline Total phosphorus & 0.63 & 0.52 & 0.52 \\
\hline
\end{tabular}

${ }^{\text {a } T h e ~ P r e m i x ~ p r o v i d e s ~ p e r ~ k g ~ o f ~ d i e t: ~ F e ~} 75$ mg, Cu 10 mg, Zn 95 mg, Mn 110mg, I 0.35mg, Se 0.30mg, $V_{A} 9,600 I U, V_{D 3} 2,700 I U, V_{E} 36 m g, V_{k 3} 3$ mg, $\mathrm{V}_{\mathrm{B} 1} 3 \mathrm{mg}, \mathrm{V}_{\mathrm{B} 2} 10.5 \mathrm{mg}, \mathrm{V}_{\mathrm{B} 6} 4.20 \mathrm{mg}, \mathrm{V}_{\mathrm{B} 12} 0.03 \mathrm{mg}$, nicotinamide 60mg, D-calcium pantothenate 18mg, folic acid 1.5mg, D-biotin 0.225 mg, choline, $500 \mathrm{mg}$.

${ }^{\mathrm{b}} \mathrm{ME}$ is a calculated value; other nutrient levels are measured values.

${ }^{\mathrm{c}}$ DDGS is distillers dried grains with solubles.

Table 2 Effect of stocking density on immune status in trachea of finishing broilers 


\begin{tabular}{cllllll}
\hline \multirow{2}{*}{ Items } & \multicolumn{3}{c}{ Stocking Density $\left(\right.$ birds $\left./ \mathrm{m}^{2}\right)$} & \multirow{2}{*}{ SEM } \\
\cline { 2 - 6 } & 8 & 10 & 12 & 14 & 16 & \\
\hline IL-1B (ng/g) & $0.0975^{\mathrm{c}}$ & $0.0993^{\mathrm{c}}$ & $0.1199^{\mathrm{b}}$ & $0.1243^{\mathrm{b}}$ & $0.1487^{\mathrm{a}}$ & 0.0038 \\
\hline IL-4 (ng/g) & 0.469 & 0.484 & 0.454 & 0.479 & 0.460 & 0.0628 \\
\hline IL-10 (ng/g) & $0.298^{\mathrm{b}}$ & $0.296^{\mathrm{b}}$ & $0.303^{\mathrm{ab}}$ & $0.334^{\mathrm{ab}}$ & $0.347^{\mathrm{a}}$ & 0.0076 \\
\hline
\end{tabular}

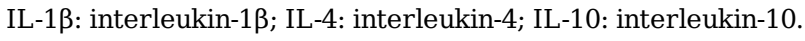

a, b, c Means within a row with not common superscripts significantly differ $(p<0.05)$.

SEM: Standard Error of Mean

Table 3 Serum differential metabolites between $16 \mathrm{birds} / \mathrm{m}^{2}$ and $10 \mathrm{birds} / \mathrm{m}^{2}$ treatment groups.

\begin{tabular}{|c|c|c|c|c|}
\hline \multirow[t]{3}{*}{ Items } & \multicolumn{2}{|l|}{ Mean } & \multirow[t]{3}{*}{ VIP } & \multirow[t]{3}{*}{$P$} \\
\hline & \multicolumn{2}{|c|}{ Stocking Density (birds $/ \mathrm{m}^{2}$ ) } & & \\
\hline & 16 & 10 & & \\
\hline 6-Phosphogluconic Acid & $6.34 \mathrm{E}-04$ & 2.05E-03 & 3.04 & 0.002 \\
\hline Threonic Acid & 2.05E-02 & $1.52 \mathrm{E}-02$ & 2.52 & 0.004 \\
\hline Xylitol & 2.08E-02 & 1.73E-02 & 2.17 & 0.025 \\
\hline Benzoic Acid & $1.62 \mathrm{E}-01$ & 3.01E-01 & 2.08 & 0.021 \\
\hline Heptadecanoic Acid & 6.64E-04 & $1.05 \mathrm{E}-03$ & 2.01 & 0.041 \\
\hline Threonine 2 & $1.78 \mathrm{E}-02$ & $5.65 \mathrm{E}-03$ & 1.99 & 0.050 \\
\hline Halostachine 2 & $2.48 \mathrm{E}-03$ & $1.75 \mathrm{E}-03$ & 1.96 & 0.030 \\
\hline Indole-3-Acetic Acid & $1.89 \mathrm{E}-04$ & 5.37E-04 & 1.95 & 0.026 \\
\hline Dioctyl Phthalate & $3.40 \mathrm{E}-03$ & $5.18 \mathrm{E}-03$ & 1.90 & 0.039 \\
\hline Salicin & $1.21 \mathrm{E}-03$ & 3.69E-03 & 1.84 & 0.000 \\
\hline Uric Acid & $1.02 \mathrm{E}-03$ & 2.35E-03 & 1.84 & 0.046 \\
\hline 2,3-Dihydroxypyridine & $2.40 \mathrm{E}-03$ & $1.53 \mathrm{E}-03$ & 1.78 & 0.048 \\
\hline Conduritol b Epoxide 2 & 4.85E-01 & $2.71 \mathrm{E}-01$ & 1.59 & 0.015 \\
\hline Glucoheptonic Acid 1 & 7.61E-03 & 2.69E-03 & 1.50 & 0.044 \\
\hline 3-(1-Pyrazolyl)-L-Alanine & $3.25 \mathrm{E}-03$ & $1.94 \mathrm{E}-03$ & 1.35 & 0.005 \\
\hline Cystine & $9.44 \mathrm{E}-03$ & $5.56 \mathrm{E}-03$ & 1.21 & 0.015 \\
\hline 4-Acetamidobutyric Acid 2 & 2.61E-03 & $1.82 \mathrm{E}-03$ & 1.14 & 0.049 \\
\hline
\end{tabular}

*VIP, variable importance in the projection

Table 4 Pathway analysis for $16 \mathrm{birds} / \mathrm{m}^{2}$ and $10 \mathrm{birds} / \mathrm{m}^{2}$ treatment groups using MetaboAnalyst 


\begin{tabular}{llll}
\hline Pathway name & Total $^{\mathrm{a}}$ & $\mathrm{Hits}^{\mathrm{b}}$ & Raw \\
\end{tabular}

\begin{tabular}{lcclll}
\hline Valine, leucine and isoleucine biosynthesis & 10 & 1 & 0.067189 & 0 \\
\hline Pentose and glucuronate interconversions & 17 & 1 & 0.1118 & 0.08333 \\
\hline Pentose phosphate pathway & 20 & 1 & 0.13033 & 0.08333 \\
\hline Cysteine and methionine metabolism & 27 & 1 & 0.17225 & 0 \\
\hline Glycine, serine and threonine metabolism & 33 & 1 & 0.20673 & 0.04786 \\
\hline Tryptophan metabolism & & & & \\
\hline Arginine and proline metabolism & 37 & 1 & 0.22901 & 0 \\
\hline Aminoacyl-tRNA biosynthesis & 38 & 1 & 0.23449 & 0 \\
\hline Purine metabolism & 44 & 1 & 0.26665 & 0 \\
\hline
\end{tabular}

${ }^{a}$ Represents the total number of metabolites in the corresponding pathway. ${ }^{b}$ Represents the actually matched number of metabolites. ${ }^{c}$ Represents the $\mathrm{P}$ values from enrichment analysis. ${ }^{\mathrm{d}}$ Represents the impact value calculated from pathway topology analysis.

\section{Figures}




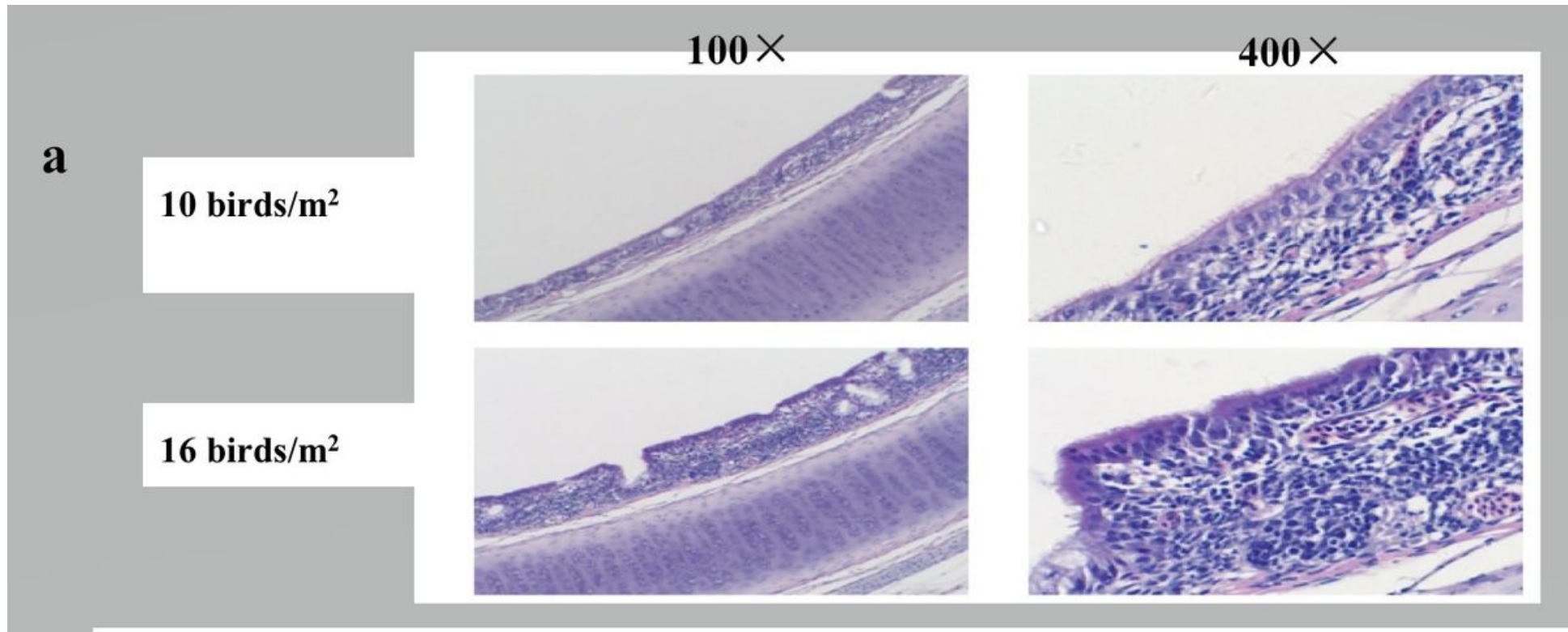

b

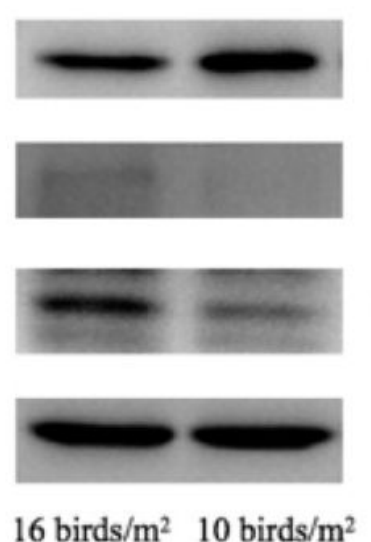

\section{claudin 1 \\ muc2}

caspase 3

actin

c

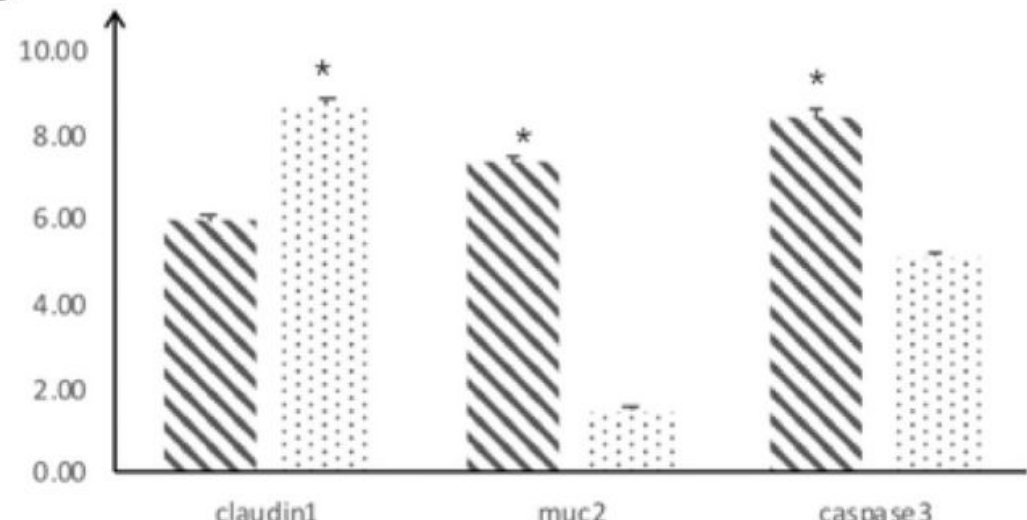

、16 birds per square metre $\quad$ :10 birds per square metre

\section{Figure 1}

Effect of stocking density in trachea on finishing broilers. (a) Representative H\&E-stained images; (b) Expression of claudin1, muc2 and caspase 3 in trachea tissue were determined by western blot. (c) Relative changes in the density of claudin 1 , muc2 and caspase3 were analyzed. Data presented as relative band intensity of claudin 1 , muc 2 and caspase 3 to $\beta$-actin. Values are means, with their standard deviations represented by vertical bars. $(* P<0.05)$. 


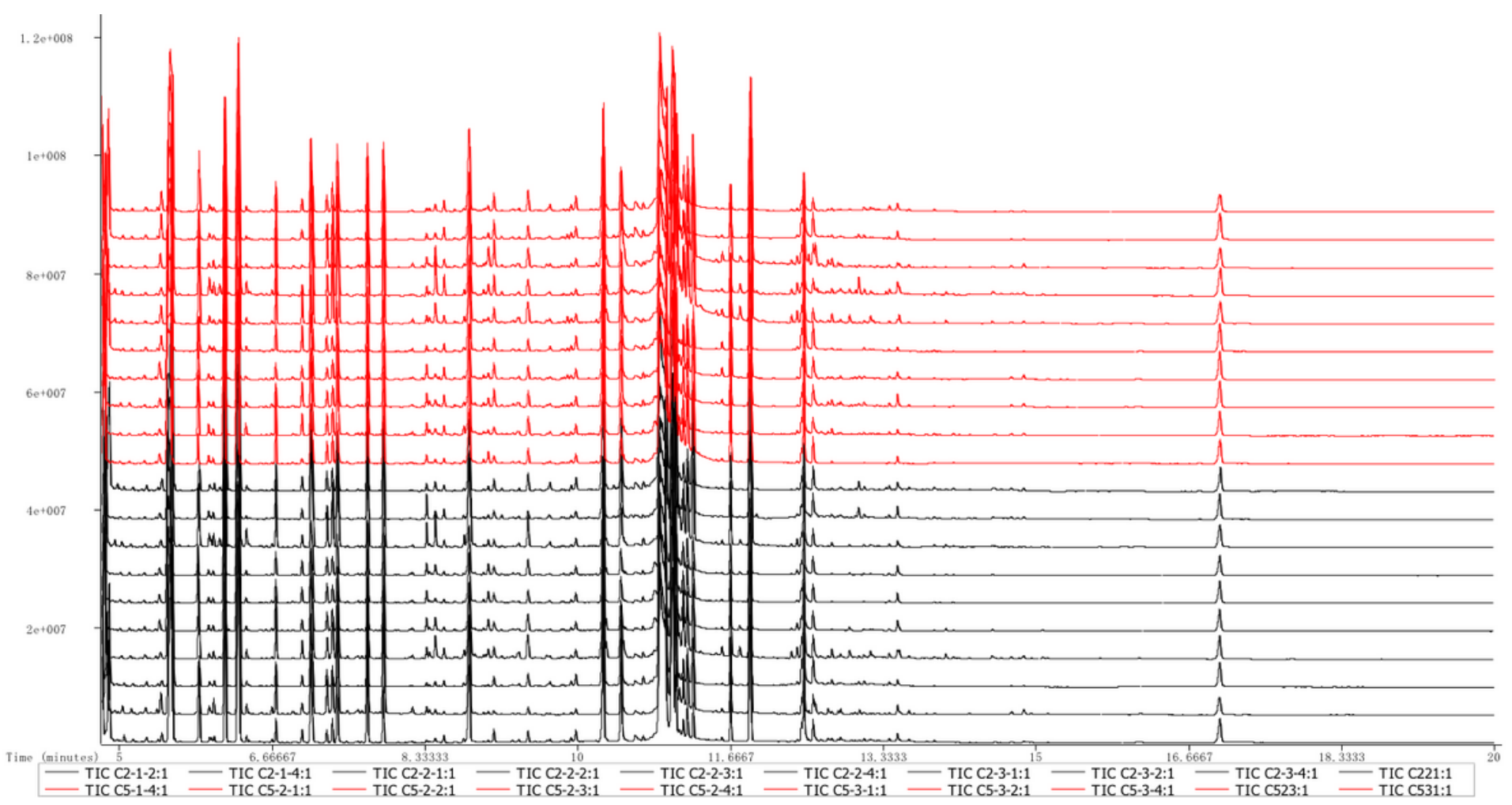

Figure 2

Total GC-TOFMS ion flow about metabolites in the broilers' serum

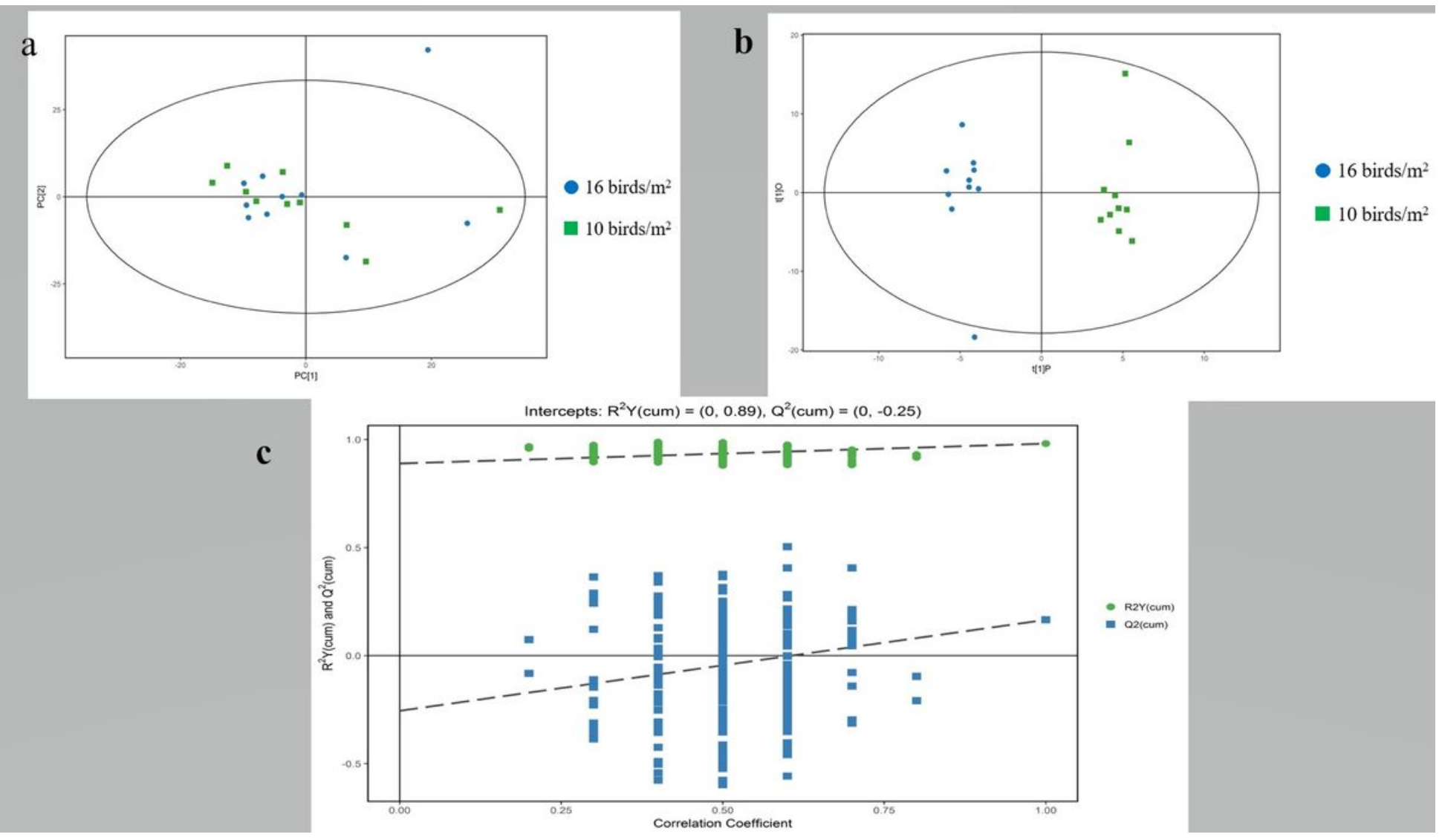

Figure 3 
(a) Principal component analysis (PCA) score plots, (b) orthogonal projections to latent structurediscriminant analysis (OPLS-DA) score plots $(\mathrm{R} 2 \mathrm{X}=0.0, \mathrm{R} 2 \mathrm{Y}=0.089, \mathrm{Q} 2=-0.25)$ and (c) permutation test of OPLS-DA from the LC-TOF/MS metabolite profiles of plasma for 16 birds / $\mathrm{m} 2 \mathrm{vs}$. group 10 birds per m2. blue circle: 16 birds per m2; green square: 10 birds per m2; Green circle: R2; blue square: Q2. The dash line represents the regression line for R2 and Q2.

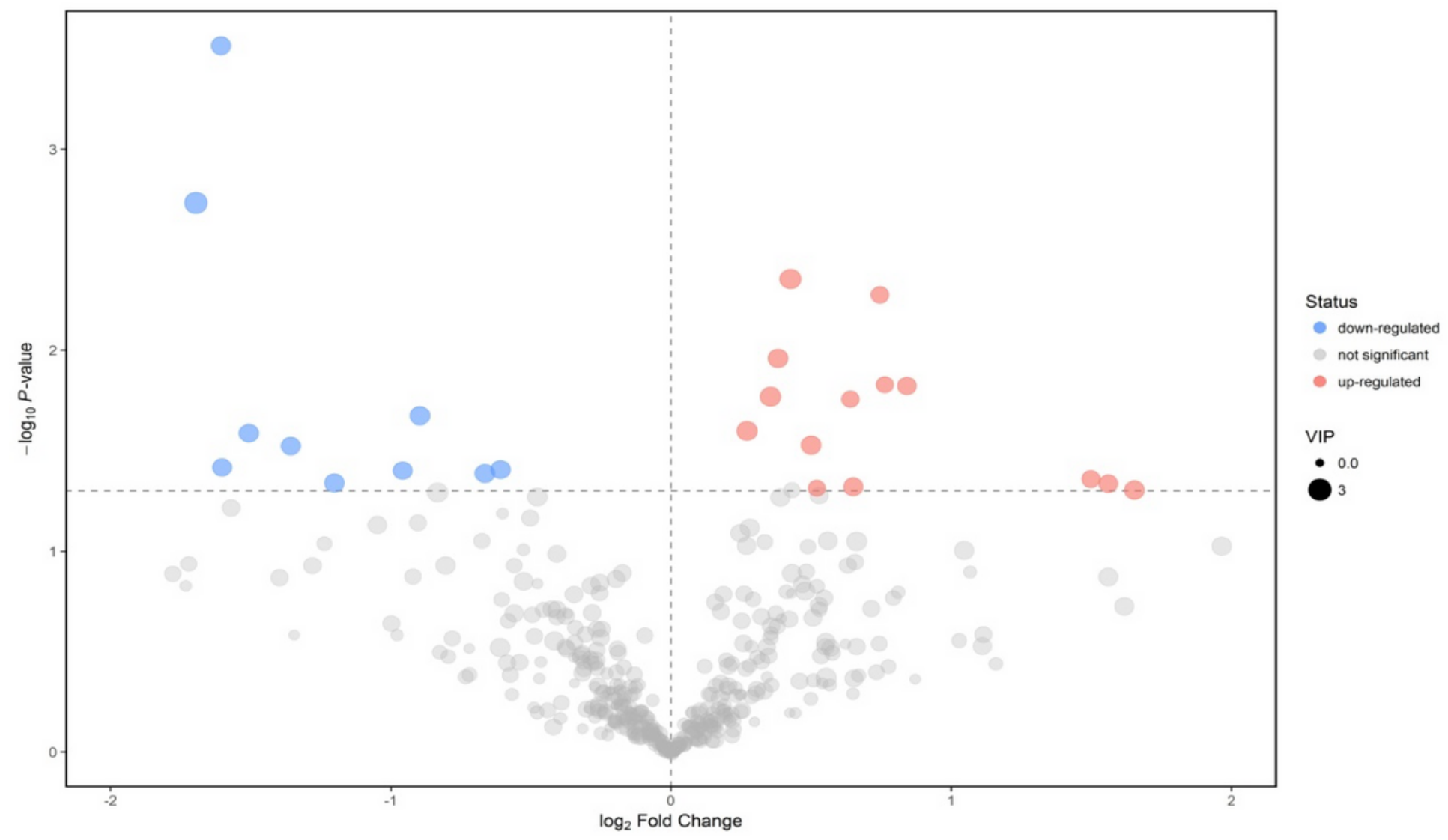

\section{Figure 4}

Volcano plots of metabolites in serum between 16 birds per $\mathrm{m} 2$ and 10 birds per $\mathrm{m} 2$ treatment groups. Each dot represents a metabolite. The larger dots indicate higher variable importance in the projection (VIP) values. The abscissa and ordinate represent the fold change and P-value of biomarkers, respectively. The increased and decreased $(P<0.05)$ biomarkers in the treatment group are represented by the red and blue dots, respectively, while the gray dots represent the unchanged $(P>0.05)$ metabolites between these two groups. 


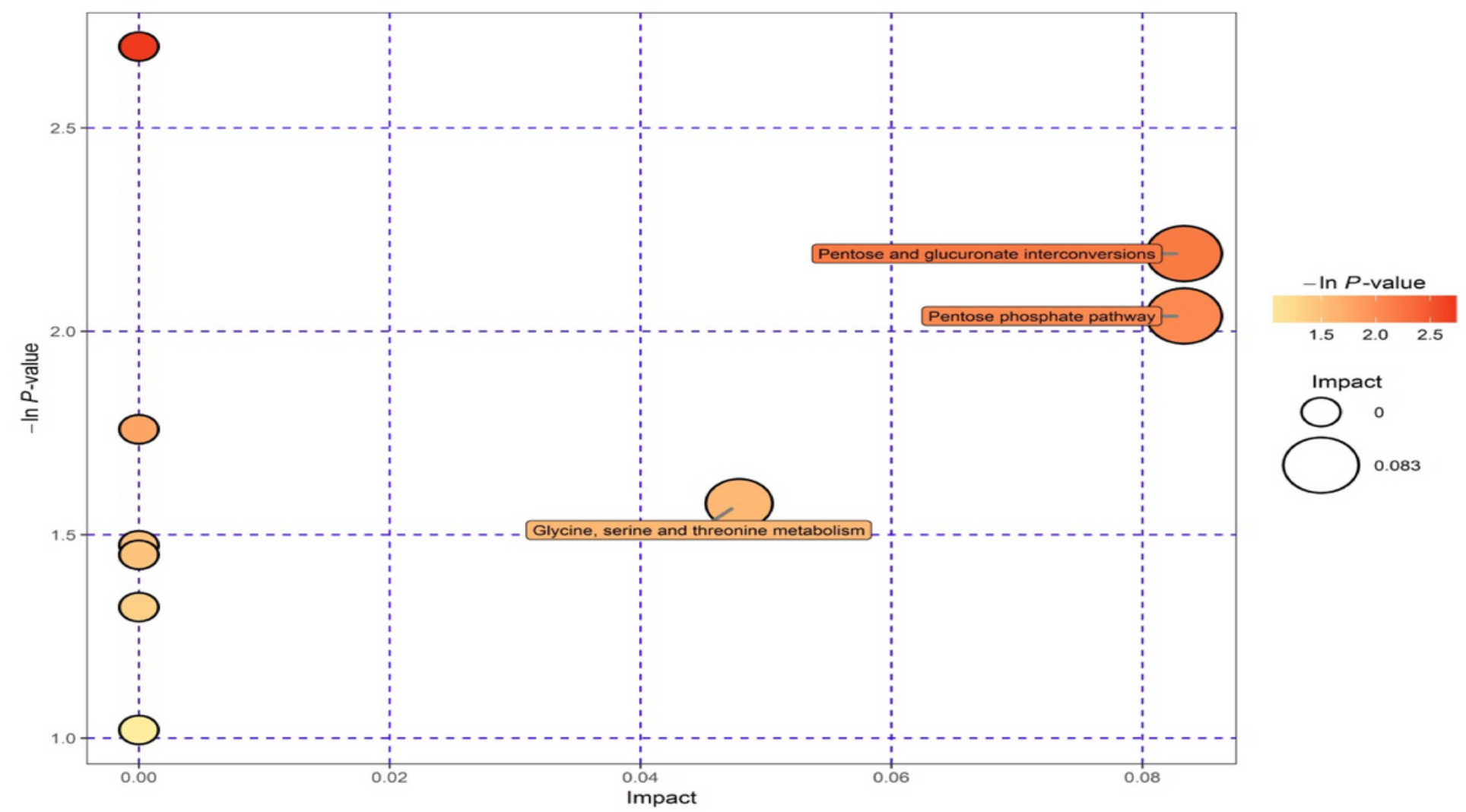

Figure 5

Graphic summary of pathway analysis between 16 birds per m2and 10 birds per $\mathrm{m} 2$ treatment groups using MetaboAnalyst. The X-axis indicates the pathway impact, and Y-axis represents the pathway enrichment. Larger sizes and darker colors indicate higher pathway enrichment and higher pathway impact values, respectively. 